Lina Lindegren*, Andrea Stuart, Marie Carlsson Fagerberg and Karin Källén

\title{
Retrospective study of maternal and neonatal outcomes after induction compared to spontaneous start of labour in women with one previous birth in uncomplicated pregnancies $\geq 41^{+3}$
}

https://doi.org/10.1515/jpm-2020-0312

Received July 6, 2020; accepted July 27, 2020; published online

August 24, 2020

\section{Abstract}

Objectives: To study the association between induction and outcome among two-parous women in uncomplicated pregnancies $\geq 41^{+3}$, stratified by first labour delivery mode and conditions present at first delivery.

Methods: The Swedish Medical Birth Register was used to identify 58,964 uncomplicated singleton pregnancies among women with one previous birth between 1998 and 2014. Women with any registered pregnancy complications were excluded to minimise the risk for indication bias. The outcomes considered were emergency caesarean section (CS), and poor neonatal outcome (Apgar score $<7$ at $5 \mathrm{~min}$, neonatal death, or meconium aspiration).

Results: Women who were induced at their second labour had higher emergency CS rates compared to women in spontaneously started deliveries (adjusted risk ratio, ARR: 2.11; 95\% CI: 2.00-2.23). Low Apgar score was more common after induction compared to spontaneously started labours (1.0 vs. 0.7\%) (ARR: 1.44; 95\% CI: 1.18-1.77). Increased CS rates were also found when comparing induction at $41+3$ to $41+6$ weeks to labour at 42 weeks or more, regardless of labour start (ARR 1.39; 95\% CI: 1.261.52).

*Corresponding author: Lina Lindegren, Institution of Clinical Sciences, Department of Obstetrics and Gynaecology, University of Lund, BMC F12, 22184 Lund Sweden; and Department of Obstetrics and Gynaecology, Helsingborg Hospital, Charlotte Yhlens gata 10, 254 37 Helsingborg Sweden, E-mail: lina.lindegren@med.lu.se. https:// orcid.org/0000-0002-5910-3907

Andrea Stuart, Helsingborg Hospital, Charlotte Yhlens gata 10, 25437 Helsingborg Sweden

Marie Carlsson Fagerberg, Ystad Hospital, Kristianstadsvägen 3, 271 33 Ystad Sweden

Karin Källén, Institution of Clinical Sciences, Department of Obstetrics and Gynaecology, University of Lund, BMC F12, 22184 Lund Sweden
Conclusions: We found an increased risk of CS and poor neonatal outcome after second labour induction in prolonged pregnancies. The second labour vaginal success rate after induction was highly dependent, on first labour delivery mode, but also on diagnoses and conditions present at the first delivery.

Keywords: caesarean section; delivery mode; induction; late term pregnancy; neonatal morbidity; parity; post-term pregnancy.

\section{Introduction}

A systematic Cochrane review (June 2018) reported significantly lower risk of adverse neonatal outcome in induced pregnancies as compared to expected management among pregnancies at or beyond term [1]. These results were confirmed in a recent randomised controlled trial (RCT) [2], and partly confirmed in another RCT [3] where significantly lower perinatal mortality rates were found in the induction group compared to the expectant management group.

We have previously reported an improved neonatal outcome among infants to primiparous women who were giving birth in delivery units with a more active management in prolonged pregnancies $\left(\geq 41^{+3}\right)$ compared to women giving birth in delivery units with less active management [4]. Among the children of multiparous women, no such association between improved neonatal outcome and active management was identified. Women, both primiparous and multiparous, who were giving birth in hospitals with active management in prolonged pregnancies were more likely to be delivered by caesarean section (CS) than were women who were giving birth in units with a more expectant management.

The above-mentioned Cochrane review reported significantly lower CS rates among induced pregnancies at or beyond term compared to pregnancies that were managed expectantly [1]. Two recent RCTs show no 
increase in CS rate after induction [2, 3], both excluded women with previous CS, and neither of them were designed to stratify their results by parity. The number of studies considering parity when evaluating induction in low-risk pregnancies at or beyond term is limited.[5] found a significant association between induction and emergency CS in low-risk pregnancies among primiparous women, but not among multiparous women [5]. Contradictory to those results, reported a significantly higher risk of emergency CS in low risk pregnancies among multiparous women after induction, compared to spontaneous start of labour [6, 7].

The objective of the current study was to explore if information regarding conditions present at the first delivery could be used to estimate the chance of a second delivery induction resulting in a vaginal delivery of a healthy child. Therefore, the association between induction and neonatal and maternal outcome among twoparous women in uncomplicated pregnancies $\geq 41^{+3}$, were studied, stratified by first labour mode of delivery and conditions present.

\section{Materials and methods}

Women with one previous delivery were identified from the Swedish Medical Birth Register (MBR), containing information from $97 \%$ of all pregnancies resulting in a delivery since 1973 [8]. All women in Sweden are offered free antenatal care. In gestational week 17-19 a free ultrasound examination is offered to determine gestational age, multiple births and severe foetal malformations. The MBR register holds information on, for example, maternal age, weight, smoking habits, body mass index (BMI), parity, start of delivery (induction, spontaneous, or elective CS), and mode of delivery (vacuum extraction [VE], forceps, or emergency CS).

Inclusion criteria were singleton prolonged pregnancies $\left(\geq 41^{+3}\right)$ and foetus in cephalic presentation among women with one previous birth. The first birth took place after 1998, and the second delivery (the index pregnancy, $n=58,964$ ) took place during the study period 19992014.

Exclusion criteria for the index delivery (second birth) were: prepregnancy diabetes, gestational diabetes, gestational hypertension, pre-eclampsia or eclampsia, placenta praevia, ablatio placentae, intrauterine death, multiple births, other presentations than cephalic, or severe foetal growth restriction (<-3 SD from the expected birth weight according to the Swedish growth standard curve [9]). Elective CS was defined as CS performed before contractions, whereas all CSs following a trial of labour were considered emergency CS. The case selection and exclusions are summarised and visualised in Figure 1.

First delivery conditions were grouped by start of delivery (spontaneous, elective CS, or induction), mode of delivery (elective CS, emergency CS, vaginal), and the indications for first delivery CS (if appropriate). The first delivery CS indications/conditions considered were: gestational diabetes, pre-eclampsia, multiple birth, noncephalic presentation, placenta praevia, preterm birth, small for gestational age (SGA), polyhydramnios, large for gestational age (LGA), post term, failed induction, dystocia and foetal asphyxia. Each woman can be assigned to one, two or more indications. The procedure has previously been used and published by Fagerberg et al. [10].

The main outcomes considered were mode of delivery (vaginal or CS), and neonatal outcome measured as a composite of Apgar $<7$ at $5 \mathrm{~min}$, meconium aspiration and neonatal death. For the main analyses, the outcome after induction was compared to that after spontaneously started labours. In sub-analyses, induction at $41^{+3}$ to $41^{+6}$ weeks was compared to the outcome among all pregnancies lasting $42^{+0}$ weeks or more, irrespective of delivery start.

\section{Statistical analyses}

All risk ratios (RR) were obtained using modified Poisson regression analyses. Adjustments were made for: year of birth (continuous, linear), maternal age (continuous, linear), BMI (continuous), and smoking (semi-continuous variable: $1=$ no smoking, $2=$ smoking $<10$ cigarettes/day, $3=$ smoking $\geq 10$ cigarettes/day). All mentioned factors were valid for the index delivery (birth number two). A sub analyses was performed which included gestational age as an independent factor, to study if the results were due to gestational age per se.

Missing data on maternal BMI and smoking were imputed with the overall means. Tests of homogeneity of the RRs across first delivery start and delivery mode were based on weighted sums of the squared deviations of the stratum specific log-RRs from their weighted means. All RRs were calculated using SPSS. Findings with $p$-values $<0.05$ were considered statistically significant.

\section{Ethical approval}

The study was approved at the Research Ethics Committee at Lund University (2015/397 2015-06-25).

\section{Results}

Table 1 presents the characteristics of the study population by start of second labour, induction or spontaneous start. The induction rate increased during the study period and increased with maternal age, BMI, and gestational duration. Women who were shorter than $150 \mathrm{~cm}$ or taller than $180 \mathrm{~cm}$ were more likely to be induced than were women of normal height (150-179 cm). Women carrying foetuses weighing $>4,500 \mathrm{~g}$ were more likely to be induced than were women with smaller foetuses, but still $72.8 \%$ of these deliveries started spontaneously.

Table 2 shows the rate of emergency CS in relation to first labour delivery mode. Overall, the CS-rate after induction was $13.6 \%$, which was more than twice the corresponding CS-rate after spontaneous start (6.1\%). The overall RR for emergency CS, induction vs. spontaneous start was 2.22. Adjustment for maternal characteristics did not 


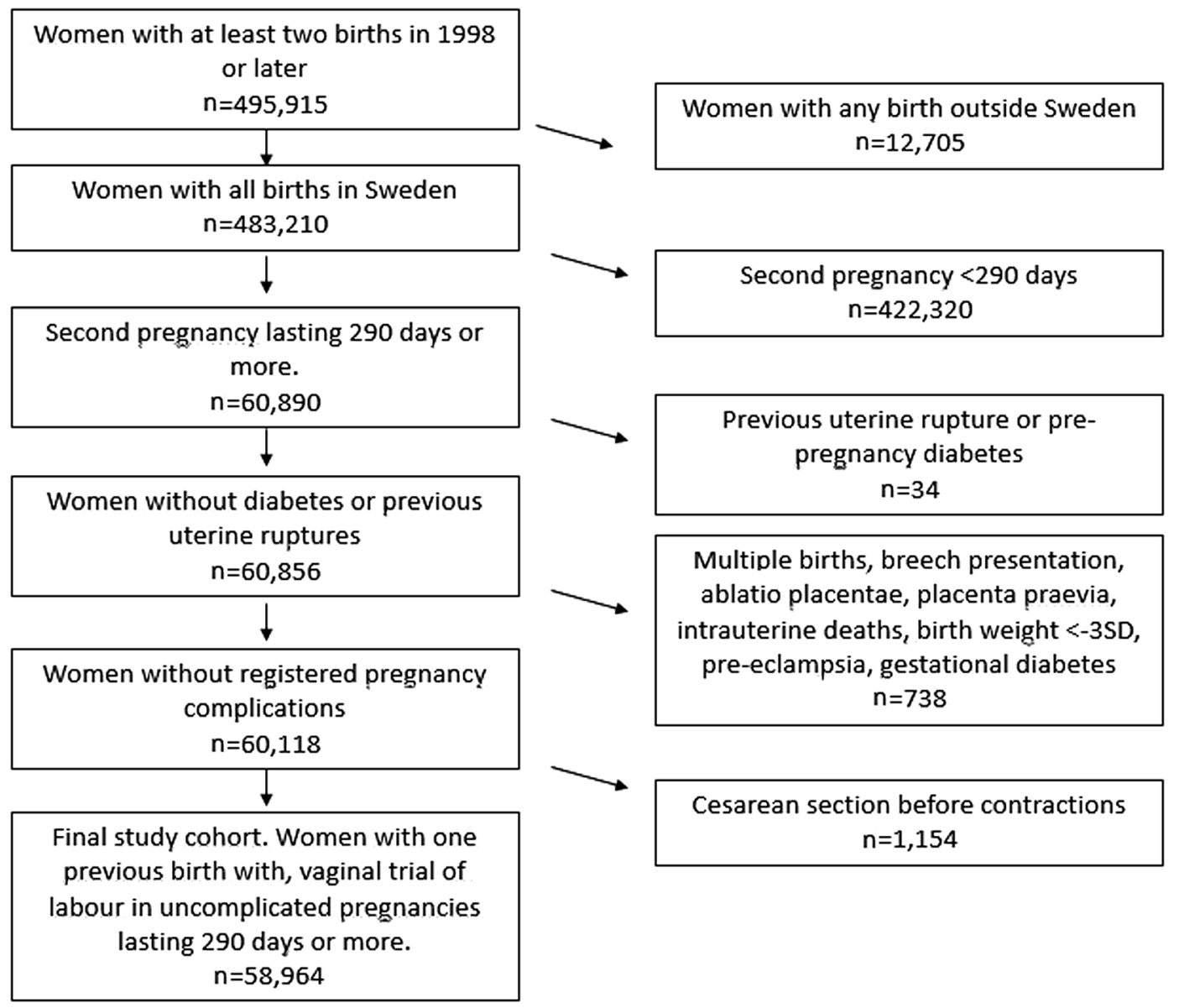

Figure 1: Flowchart: data catchment and selection criteria for formation of the final study population.

substantially change the RR (ARR 2.11). Adjustment for gestational duration in addition to the mentioned covariates lowered the ARR marginally (data not shown). The emergency CS-rate after induction ranged from 5.1\% among women with one previous vaginal delivery to $52.1 \%$ among women with one previous emergency CS. Even though the CS-rate varied considerably depending on first labour delivery mode, an increased ARR for emergency CS after induction was found within each group of two-parous women, divided by first labour delivery mode. There was no significant impact of first delivery onset (induction or spontaneous) on the CS-rate after second delivery induction (data not shown).

Figure 2 shows the emergency CS rate by second labour onset (spontaneous or induction) among women who had one previous CS. The CS rates are stratified by conditions present at, or indication for, the first delivery CS. The corresponding numbers are shown in supplementary table $\mathrm{S}$. The figure shows that the second labour CS-rate after induction varies considerably with the indication for the first labour CS. The highest CS rates after induction (above 50\%) were noted among women who had a previous CS with an indication of post term pregnancy, failed induction, dystocia, or foetal asphyxia. Regardless of conditions/indications present at first CS, the second labour emergency CS rate was higher after induction than after spontaneous onset.

Table 3 shows the association between the second delivery start and poor neonatal outcome, measured as Apgar score $<7$ at $5 \mathrm{~min}$, meconium aspiration, neonatal death, or as a composite variable including these three mentioned outcomes. Children to women with a previous vaginal birth were at lower risk of poor neonatal outcome than were children of women with one previous CS, both after induction and after spontaneous onset of second labour. A poor neonatal outcome was found for $1.1 \%$ of the children born after induced labour, compared to $0.8 \%$ after spontaneously started births (RR 1.41). The association between induction and poor neonatal outcome was marginally altered after adjustment for possible confounders (ARR 1.37). Irrespective of the first labour delivery mode, an association between second labour induction and poor neonatal outcome was indicated, but not statistically significant. 
Table 1: Start of second labour in uncomplicated* pregnancies lasting $\geq 41^{+3}$ days, by maternal and foetal characteristics among women with one previous birth.

\begin{tabular}{|c|c|c|c|c|c|c|}
\hline \multirow[t]{3}{*}{ Characteristics, second labour } & \multirow{3}{*}{$\begin{array}{r}\text { Total } \\
\mathbf{n}\end{array}$} & & & \multicolumn{2}{|c|}{ Start of second labour } & \multirow[t]{3}{*}{ p-Value $X^{2}$} \\
\hline & & \multicolumn{2}{|c|}{ Induction, $n=13,330$} & \multicolumn{2}{|c|}{$\begin{array}{r}\text { Spontaneous, } \\
n=45,634\end{array}$} & \\
\hline & & $\mathrm{n}$ & $\%$ & $\mathbf{n}$ & $\%$ & \\
\hline Year of delivery & & & & & & $<0.001$ \\
\hline 1999-2004 & 14,727 & 2,466 & 16.7 & 12,261 & 83.3 & \\
\hline $2005-2009$ & 21,384 & 4,655 & 21.8 & 16,729 & 78.2 & \\
\hline 2010-2014 & 22,853 & 6,209 & 27.2 & 16,644 & 72.8 & \\
\hline Maternal age, years & & & & & & $<0.001$ \\
\hline$<25$ & 4,922 & 992 & 20.2 & 3,930 & 79.8 & \\
\hline $25-34$ & 41,553 & 9,090 & 21.9 & 32,463 & 78.1 & \\
\hline$\geq 35$ & 12,489 & 3,248 & 26.0 & 9,241 & 74.0 & \\
\hline Body mass index, $\mathrm{kg} / \mathrm{m}^{2}$ & & & & & & $<0.001$ \\
\hline$<18.5$ & 745 & 118 & 15.8 & 627 & 84.2 & \\
\hline $18.5-29.9$ & 46,021 & 10,070 & 21.9 & 35,951 & 78.1 & \\
\hline$\geq 30$ & 7,177 & 2,101 & 29.3 & 5,076 & 70.7 & \\
\hline Not known & 5,021 & 1,041 & 20.7 & 3,980 & 79.3 & \\
\hline Maternal height, $\mathrm{cm}$ & & & & & & 0.039 \\
\hline$<150$ & 77 & 22 & 28.6 & 55 & 71.4 & \\
\hline $150-179$ & 54,407 & 12,296 & 22.6 & 42,111 & 77.4 & \\
\hline$\geq 180$ & 1,724 & 425 & 24.7 & 1,299 & 75.3 & \\
\hline Not known & 2,756 & 587 & 21.3 & 2,169 & 78.7 & \\
\hline Maternal smoking & & & & & & 0.061 \\
\hline Smoking & 2,793 & 658 & 23.6 & 2,135 & 76.4 & \\
\hline Non-smoking & 53,477 & 12,107 & 22.6 & 41,370 & 77.4 & \\
\hline Not known & 2,694 & 565 & 21.0 & 2,129 & 79.0 & \\
\hline Birth weight, g & & & & & & $<0.001$ \\
\hline$<2,500$ & 91 & 12 & 13.2 & 79 & 86.8 & \\
\hline $2,500-4,500$ & 51,529 & 11,324 & 22.0 & 40,205 & 78.0 & \\
\hline$>4,500$ & 7,344 & 1,994 & 27.2 & 5,350 & 72.8 & \\
\hline Interval between first and second deliveries & & & & & & $<0.001$ \\
\hline$<18$ months & 2,780 & 546 & 19.6 & 2,234 & 80.4 & \\
\hline$\geq 18$ months & 56,184 & 12,784 & 22.8 & 43,400 & 77.2 & \\
\hline Gestational duration, weeks & & & & & & $<0.001$ \\
\hline 41 & 35,210 & 3,196 & 9.1 & 32,014 & 90.9 & \\
\hline 42 & 23,016 & 9,778 & 42.5 & 13,238 & 57.5 & \\
\hline$\geq 43$ & 738 & 356 & 48.2 & 382 & 51.8 & \\
\hline
\end{tabular}

${ }^{a}$ Complications excluded were: Women with pre-pregnancy diabetes, gestational diabetes, preeclampsia, gestational hypertension, previous uterine rupture, ablatio placentae, placenta praevia, intrauterine death, serious intrauterine growth restriction ( $<-3$ standard deviations corresponding to lowest 1.3 per thousand births), multiple births, and other presentations than cephalic.

Tables $4 \mathrm{~A}$ and $4 \mathrm{~B}$ shows the outcome after induction at $41^{+3}$ to $41^{+6}$ weeks compared to the outcome among all labours at $42^{+0}$ weeks or more, irrespective of induction or spontaneous start. Table 4A shows that emergency CS was significantly more common in induced labours at $41^{1+3}$ to $41^{16}$ than in labours occurring at 42 weeks or more. Adjustment for maternal characteristics altered the risk estimates marginally (ARR: 1.39; 95\% CI: 1.26-1.52). No significantly increased risk was found for poor neonatal composite outcome, induced labour at $41^{+3}$ to $41^{+6}$ weeks vs. all labours at $42^{+0}$ weeks or more.

\section{Discussion}

Our study population consisted of women with one previous birth (vaginal or CS), with uncomplicated second pregnancies lasting $41^{+3}$ weeks or more. By excluding all pregnancies with any medical indication for induction (other than prolonged pregnancy), our intention was to evaluate the impact of induction per se on maternal and foetal outcome. A significant association between second labour induction and emergency CS was found. The success rate (rate of vaginal birth) after induction was 
Table 2: Risk of emergency CS in second labour among uncomplicated pregnancies lasting $\geq 41^{+3}$ days, in relation to start of second labour, by first labour start and delivery mode. Includes women with one previous birth with second delivery trial of labour. Singleton, cephalic presentation only.

\begin{tabular}{|c|c|c|c|c|c|c|c|c|}
\hline \multirow[b]{4}{*}{ First delivery } & \multicolumn{6}{|c|}{ Start of second labour } & \multirow{2}{*}{\multicolumn{2}{|c|}{$\begin{array}{l}\text { RR for emergency CS second labour, } \\
\text { induction vs. spontaneous start }\end{array}$}} \\
\hline & \multicolumn{3}{|c|}{ Induction } & \multicolumn{3}{|c|}{ Spontaneous start } & & \\
\hline & \multicolumn{2}{|c|}{ Caesarean } & \multirow{2}{*}{$\frac{\text { Total }}{n}$} & \multicolumn{2}{|c|}{ Caesarean } & \multirow{2}{*}{$\frac{\text { Total }}{n}$} & \multirow{2}{*}{$\begin{array}{r}\text { RR } \\
5 \% \mathrm{Cl}) \\
\end{array}$} & \multirow{2}{*}{$\begin{array}{r}\text { ARR } \\
\text { RR }(95 \% \mathrm{Cl})\end{array}$} \\
\hline & $\mathbf{n}$ & (\%) & & $\mathbf{n}$ & (\%) & & & \\
\hline Vaginal delivery & 543 & $(5.1)$ & 10,616 & 863 & $(2.2)$ & 39,530 & $2.34(2.11-2.60)$ & $2.26(2.03-2.51)$ \\
\hline Emergency CS & 908 & $(52.1)$ & 1,744 & 1,556 & $(37.7)$ & 4,131 & $1.38(1.30-1.47)$ & $1.36(1.28-1.44)$ \\
\hline Elective CS & 363 & $(37.4)$ & 970 & 380 & $(19.3)$ & 1,973 & $1.94(1.72-2.19)$ & $1.85(1.64-2.09)$ \\
\hline Total, regardless of first labour delivery mode & 1,814 & $(13.6)$ & 13,330 & 2,799 & $(6.1)$ & 45,634 & $2.22(2.10-2.35)$ & $2.11(2.00-2.23)$ \\
\hline
\end{tabular}

${ }^{a}$ Complications excluded were: women with pre-pregnancy diabetes, gestational diabetes, preeclampsia, gestational hypertension, previous uterine rupture, ablatio placentae, placenta praevia, intrauterine death, serious intrauterine growth restriction ( $<-3$ standard deviations corresponding to lowest 1.3 per thousand births), multiple births, and other presentations than cephalic. ARR=RR adjusted for year of birth (index pregnancy), maternal age, BMI, and smoking.

influenced by the first labour delivery mode and first CS indications or medical conditions present. Adjustment for possible confounders only marginally altered the risk estimates. Furthermore, we found a significantly increased risk of emergency CS among induced labours at $41^{+3}$ to $41^{+6}$ weeks compared to labours (irrespective of induction or spontaneous start) in pregnancies lasting $42^{+0}$ weeks or more.

We have previously reported that infants to multiparous women did not gain from "active obstetric management" in prolonged or post term pregnancies compared to less active management. In the current study, infants of women who were induced were actually at higher risk of poor neonatal outcome than were infants of mothers with spontaneous start of labour. However, when comparing neonatal outcome after induction at $41^{+3}$ to $41^{+6}$ weeks with the outcome after labours in pregnancies that continued to 42 weeks or beyond, no increased risk for poor neonatal outcome in the induction group was detected. Our results indicate that a thorough medical history should be taken before deciding on induction for prolonged second pregnancies, given that no other medical indication for induction is present.

The present results are not in line with the latest Cochrane report showing that active management with induction in prolonged pregnancies decreases the CS rate and improves neonatal outcome [1]. The included studies varied in size, study period, and inclusion criteria (e.g., to what extent women with one prior CS were included or not). Our study is not comparable with the RCTs included in the meta-analysis, or other recent RCTs [3], since our aim was not to evaluate the type of management, but merely to

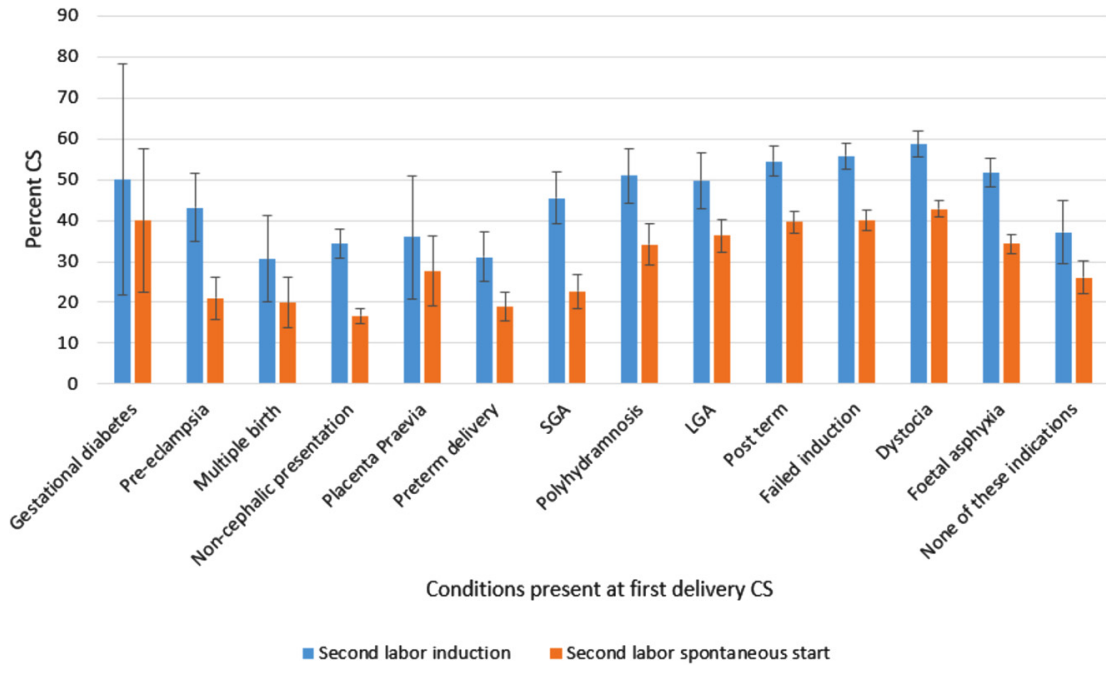

Figure 2: Risk of emergency CS in second labour among uncomplicated pregnancies lasting $\geq 41^{+3}$ days, in relation to start of second labour among women with one previous CS, by conditions present at first delivery CS. Includes women with second delivery trial of labour after CS. Singleton, cephalic presentation only.

Complications excluded were: women with pre-pregnancy diabetes, gestational diabetes, previous uterine rupture, ablatio placentae, placenta praevia, intrauterine death, gestational hypertension, preeclampsia, eclampsia, serious intrauterine growth restriction ( $<-3$ standard deviations corresponding to lowest 1.3 per thousand births), multiple births, and other presentations than cephalic. Each woman can appear in more than one indication group. 
Table 3: Risk of Apgar score $<7$ at 5 min, meconium aspiration, or neonatal death among pregnancies lasting $\geq 41^{+3}$ weeks, in relation to start of second labour among infants born to women with one previous birth, by first labour start and delivery mode. Singleton, cephalic presentation only.

\begin{tabular}{|c|c|c|c|c|c|c|c|c|}
\hline \multirow[b]{4}{*}{ First delivery } & \multicolumn{6}{|c|}{ Start of second labour } & \multicolumn{2}{|c|}{$\begin{array}{r}\text { Risk ratio for neonatal outcome* } \\
\text { second labour, induction vs. spon- } \\
\text { taneous start }\end{array}$} \\
\hline & \multicolumn{3}{|c|}{ Induction } & \multicolumn{3}{|c|}{ Spontaneous start } & & \\
\hline & \multicolumn{2}{|c|}{ Cases } & \multirow{2}{*}{$\frac{\text { Total }}{\mathrm{n}}$} & \multicolumn{2}{|c|}{ Cases } & \multirow{2}{*}{$\frac{\text { Total }}{n}$} & $\mathbf{R} \mathbf{R}$ & ARR \\
\hline & $\mathbf{n}$ & (\%) & & $\mathbf{n}$ & (\%) & & RR $95 \% \mathrm{Cl}$ & ARR $95 \% \mathrm{Cl}$ \\
\hline Apgar score $<7$ & 133 & $(1.0)$ & 13,330 & 303 & $(0.7)$ & 45,634 & $1.50(1.23-1.84)$ & $1.44(1.18-1.77)$ \\
\hline Meconium aspiration & 18 & $(0.1)$ & 13,330 & 63 & $(0.1)$ & 45,634 & $0.98(0.58-1.65)$ & $0.95(0.57-1.60)$ \\
\hline Neonatal death & 2 & $(0.0)$ & 13,330 & 18 & $(0.0)$ & 45,634 & $0.38(0.09-1.64)$ & \\
\hline Any of these (composite) & 145 & $(1.1)$ & 13,330 & 352 & $(0.8)$ & 45,634 & $1.41(1.16-1.71)$ & $1.37(1.13-1.66)$ \\
\hline \multicolumn{9}{|c|}{ Composite neonatal outcome by first labour delivery mode } \\
\hline Vaginal delivery & 72 & $(0.7)$ & 10,616 & 218 & $(0.6)$ & 39,530 & $1.23(0.94-1.60)$ & $1.20(0.92-1.57)$ \\
\hline Emergency CS & 26 & $(2.7)$ & 970 & 40 & $(2.0)$ & 1,973 & $1.18(0.84-1.67)$ & $1.17(0.83-1.65)$ \\
\hline Elective CS & 47 & $(2.7)$ & 1,744 & 94 & $(2.3)$ & 4,131 & $1.32(0.81-2.15)$ & $1.33(0.82-2.14)$ \\
\hline
\end{tabular}

$A A R=R R$ adjusted for year of birth (index pregnancy), maternal age, BMI, and smoking. ${ }^{a}$ Apgar score $<7$ at 5 min, meconium aspiration, or neonatal death.

Table 4A: Emergency CS rate in induced labours at $41^{+3}$ to $41^{+6}$ weeks compared to all labours occurring at $42^{+0}$ weeks or more. Includes women with one previous birth with second delivery trial of labour. Singleton, cephalic presentation only.

\begin{tabular}{|c|c|c|c|c|c|c|c|c|c|}
\hline & \multirow{2}{*}{$\frac{\text { Total }}{\mathbf{n}}$} & \multicolumn{2}{|c|}{ Emergency CS } & \multicolumn{2}{|c|}{ Vaginal delivery } & \multicolumn{4}{|c|}{$\begin{array}{r}\text { Risk ratio for emergency CS, induction } \\
\text { week } 41^{+3} \text { to } 41^{+6} \mathrm{vs} \text {. any labour } \geq 42^{+0} \\
\text { weeks }\end{array}$} \\
\hline & & $\mathbf{n}$ & (\%) & $\mathbf{n}$ & (\%) & $\mathbf{R R}$ & $95 \% \mathrm{Cl}$ & ARR & $95 \% \mathrm{Cl}$ \\
\hline Induction at $41^{+3}$ to $41^{+6}$ weeks & 3,196 & 460 & $(14.4)$ & 2,736 & $(85.6)$ & 1.45 & $1.32-1.59$ & 1.39 & $1.26-1.52$ \\
\hline Labour at $42^{+0}$ weeks or more, regardless of start & 23,754 & 2371 & $(10.0)$ & 21,383 & $(90.0)$ & 1.00 & Reference & 1.00 & Reference \\
\hline
\end{tabular}

$A A R=R R$ adjusted for year of birth (index pregnancy), maternal age, BMI, and smoking.

Table 4B: Risk for poor neonatal composite outcome after induced labours at $41^{+3}$ to $41^{+6}$ weeks compared to all labours occurring at $42^{+0}$ weeks or more. Includes women with one previous birth with second delivery trial of labour. Singleton, cephalic presentation only.

\begin{tabular}{|c|c|c|c|c|c|c|c|c|c|}
\hline & \multirow{2}{*}{$\underbrace{\text { n }}_{\text {Total }}$} & \multicolumn{2}{|c|}{$\begin{array}{r}\text { Emergency } \\
\text { CS }\end{array}$} & \multicolumn{2}{|c|}{ Vaginal delivery } & \multicolumn{4}{|c|}{$\begin{array}{r}\text { Risk ratio for poor neonatal composite } \\
\text { outcome*, induction week } 41^{+3} \text { to } 41^{+6} \text { vs } \\
\text { any labour } \geq 42^{+0} \text { weeks }\end{array}$} \\
\hline & & $\mathbf{n}$ & (\%) & $\mathbf{n}$ & (\%) & $\mathbf{R R}$ & $95 \% \mathrm{Cl}$ & ARR & $95 \% \mathrm{Cl}$ \\
\hline Induction at $41^{+3}$ to $41^{+6}$ weeks & 3,196 & 39 & $(1.2)$ & 3,157 & $(98.8)$ & 1.26 & $0.90-1.77$ & 1.24 & $0.88-1.73$ \\
\hline Labour at $42^{+0}$ weeks or more, regardless of start & 23,754 & 231 & $(1.0)$ & 23,523 & $(99.0)$ & 1.00 & Reference & 1.00 & Reference \\
\hline
\end{tabular}

$A A R=R R$ adjusted for year of birth (index pregnancy), maternal age, BMI, and smoking. ${ }^{a}$ Apgar score $<7$ at 5 min, meconium aspiration, or neonatal death.

observe the outcome after induction, and analyse it in relation to available information regarding the first delivery.

Previous studies have reported that pregnancies continuing to or beyond 294 days (42+ weeks) imply a significantly higher risk for stillbirths among foetuses to primiparous women compared to multiparous women [11, 12]. These results were in concordance with our previous Swedish study indicating that units with a pro-active management of prolonged pregnancies had higher CS-rates than had units with a less active management in these pregnancies [4]. 
There are limitations of this study. Even if efforts were made to exclude all pregnancies with pre-labour complications, it could not be ruled out that some complicated pregnancies nevertheless were included, and that they were likely be over-represented in the induction group. This could, to some extent, explain the high CS rate among women with induced labour. However, the current study was observational, with the focus to explore to what extent the experiences from the first delivery could be used to predict the maternal and neonatal outcome after second delivery induction compared to spontaneous start. With the advantage of the large study group used in this retrospective study, the risk estimates for the considered outcomes could be calculated with high precision.

Few reports have been published addressing the obstetric management in prolonged or post term pregnancies stratified by parity. Even if the results for primiparous and multiparous women sometimes have been reported separately, most RCTs were not dimensioned to be able to detect a significant heterogeneity between primiparous and multiparous women in relation to obstetric management in prolonged pregnancies. In a recent Swedish RCT [3], a significantly decreased risk of perinatal death was observed in pregnancies induced at $41^{+0}$ weeks compared to expectantly managed pregnancies. Despite the fact that all deaths occurred among children to primiparous women, the difference between primiparous and multiparous women did not reach significance, probably due to low numbers. Many of the RCTs excluded women with previous CS [2, 3], even though those women constitute a growing proportion of all multiparous women giving birth.

\section{Conclusions}

Evidence suggests that parity should be considered when deciding upon the obstetric management of prolonged deliveries. The need of individual assessment of advantages and disadvantages of labour induction is especially important among women with a history of prior labour complications. Those women are often excluded from recent investigations regarding obstetric management in prolonged pregnancies. The results from the present study, including uncomplicated pregnancies only, indicate a huge variation of success rate after second delivery induction, depending on first labour delivery mode, and the indication for CS when applicable. Knowledge of this variation may be important when deciding upon obstetric management of prolonged pregnancies among women with one previous birth.
Research funding: Stig and Ragna Gorthon Foundation; Thelma Zoegas Foundation.

Author contributions: All authors have accepted responsibility for the entire content of this manuscript and approved its submission.

Competing interests: Authors state no conflict of interest. Informed consent: Informed consent was obtained from all individuals included in this study.

Ethical approval: The study was approved at the Research Ethics Committee at Lund University (2015/397 2015-06-25).

\section{References}

1. Middleton P, Shepherd E, Crowther CA. Induction of labour for improving birth outcomes for women at or beyond term. Cochrane Database Syst Rev 2018;5:CD004945..

2. Keulen JK, Bruinsma A, Kortekaas JC, et al. Induction of labour at 41 weeks versus expectant management until 42 weeks (INDEX): multicentre, randomised non-inferiority trial. BMJ 2019;364:1344.

3. Wennerholm UB, Saltvedt S, Wessberg A, Alkmark M, Bergh C, Wendel SB, et al. Induction of labour at 41 weeks versus expectant management and induction of labour at 42 weeks (Swedish Postterm Induction Study, SWEPIS): multicentre, open label, randomised, superiority trial. BMJ 2019;367:16131.

4. Lindegren L, Stuart A, Herbst A, Källén K. Improved neonatal outcome after active management of prolonged pregnancies beyond $41^{+2}$ weeks in nulliparous, but not among multiparous women. Acta Obstet Gynecol Scand 2017;96:1467-74.

5. Dublin S, Lydon-Rochelle M, Kaplan RC, Watts DH, Critchlow CW. Maternal and neonatal outcomes after induction of labor without an identified indication. Am J Obstet Gynecol 2000;183:986-94.

6. Thorsell M, Lyrenäs S, Andolf E, Kaijser M. Induction of labor and the risk for emergency cesarean section in nulliparous and multiparous women. Acta Obstet Gynecol Scand 2011;90:1094-9.

7. Jacquemyn $Y$, Michiels I, Martens G. Elective induction of labour increases caesarean section rate in low risk multiparous women. J Obstet Gynaecol 2012;32:257-9.

8. Cnattingius S, Ericson A, Gunnarskog J, Källén B. A quality study of a medical birth registry. Scand J Soc Med 1990;18:143-8.

9. Marsál K, Persson PH, Larsen T, Lilja H, Selbing A. Sultan B Intrauterine growth curves based on ultrasonically estimated foetal weights. Acta Paediatr 1996;85:843-8.

10. Fagerberg MC, Maršál K, Källén K. Predicting the chance of vaginal delivery after one cesarean section: validation and elaboration of a published prediction model. Eur J Obstet Gynecol Reprod Biol 2015;188:88-94.

11. Ingmarsson I, Kallen K. Stillbirths and risk of neonatal death in 76761 postterm pregnancies in Sweden 1982-1991: a register study. Acta Obstet Gynecol Scand 1997;76:658-62.

12. Hilder L, Sairam S, Thilaganathan B. Influence of parity on fetal mortality in prolonged pregnancy. Eur J Obstet Gynecol Reprod Biol 2007;132:167-70.

Supplementary Material: The online version of this article offers supplementary material (https://doi.org/10.1515/jpm-2020-0312). 